\title{
COMMISSIONING OF THE LHC BEAM TRANSFER LINE TI 8
}

\author{
J. Uythoven, G. Arduini, B. Goddard, D. Jacquet, V. Kain, M. Lamont, V. Mertens, A. Spinks, \\ J. Wenninger (CERN, Geneva, Switzerland), Y. Chao (TJNAF, Newport News, Virginia, USA)
}

\section{Abstract}

The first of the two LHC transfer lines was commissioned in autumn 2004. Beam reached an absorber block located some $2.5 \mathrm{~km}$ downstream of the SPS extraction point at the first shot, without the need of any threading. The hardware preparation and commissioning phase will be summarised, followed by a description of the beam tests and their results regarding optics and other line parameters, including the experience gained with beam instrumentation, the control system and the machine protection equipment.

\section{INTRODUCTION}

The transfer lines between the CERN SPS and the LHC [1] are housed in new tunnels with a total length of $5.6 \mathrm{~km}$ (Fig. 1). The two lines comprise some 400 dipole magnets, 200 quadrupole magnets and 80 corrector magnets. TI 2 will transport beam from the SPS Long Straight Section (LSS) 6 to the clockwise LHC ring near interaction region (IR) 2. The upstream part of this line is installed. As the downstream part is used as transport path for LHC magnets, installation of this part and overall beam commissioning of this line will only take place just before the LHC start-up, planned for summer 2007.

The TI 8 transfer line carries beam from SPS LSS4 to the counter clockwise LHC ring near IR8. Installation of the full line up to a few metres before the LHC tunnel was completed in summer 2004. As the SPS and the LHC differ in altitude by more than $60 \mathrm{~m}$, bending takes place in both planes (Fig. 2).

The new extraction from the SPS and the first hundred meters of TI 8 (called TT40) were already commissioned with beam in autumn 2003 [2]. This paper describes the hardware commissioning period, the cold checkout and the beam tests of the subsequent, $2.5 \mathrm{~km}$ long TI 8 part which took place during the summer and autumn of 2004.

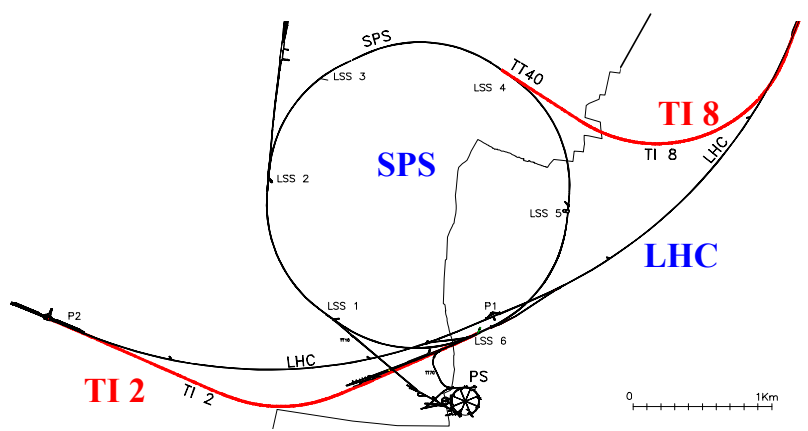

Figure 1: Layout of the LHC transfer lines TI 2 and TI 8.

\section{HARDWARE COMMISSIONING AND COLD CHECKOUT}

Immediately after the installation of the equipment in TI 8 was terminated, the hardware commissioning took place during 7 weeks. During this period the following tasks were performed:

- Adjustment and calibration of the magnet cooling water circuits and verification of all magnet temperature interlocks;

- Power tests and, if necessary, adjustment of the power converters for operation up to nominal currents;

- Verification of all magnet polarities, done by adjusting the currents from the control room by using the same software and database applications as used afterwards for beam commissioning;

- Verification of the communication with all beam instrumentation;

- Test of the vacuum system of the line (pumps, valves, gauges and interlocks);

- Test of the control system, fixed displays, logging and data base applications;

- Tests of the interlock system;

- Tests of the access system.

All tests were successfully completed, implying the polarity reversal on a number of magnets.

Several air, cooling water and magnet temperatures were monitored during prolonged periods of continuous powering of TI 8 (following the normal SPS cycle). The measurements, Fig. 3, confirm that the lines can be operated during quite extended periods, in contrast to earlier predictions. The measured maximum temperature rise of the air was about 3 degrees, indicating that the heat produced by the magnets is efficiently transported out of the tunnel by the cooling water. Only the temperature measured by the probe mounted inside the tunnel wall did

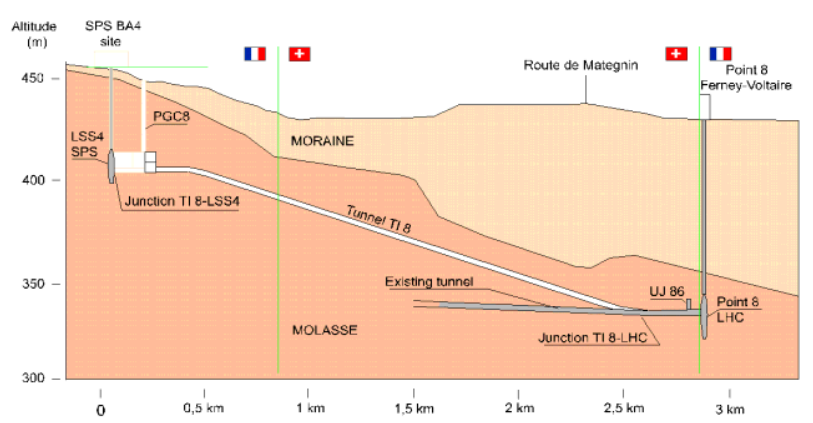

Figure 2: Vertical projection of the TI 8 transfer line. 


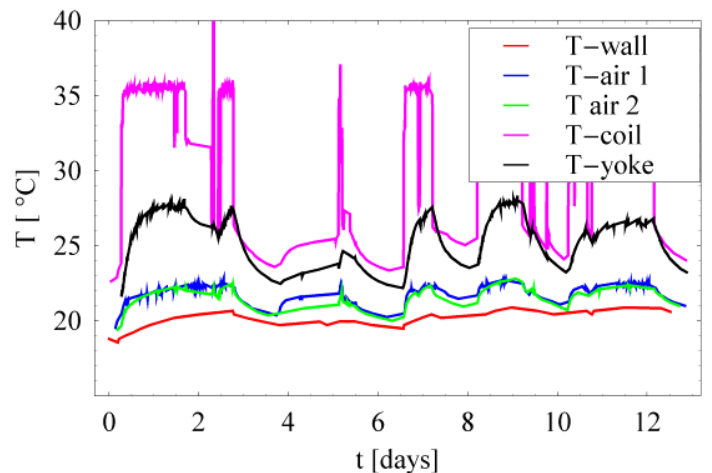

Figure 3: Measured temperatures in the TI 8 tunnel during the hardware commissioning period.

not level off yet during the test period.

The hardware commissioning was followed by two weeks of 'cold checkout'. The accent during this period was on the control system and remote operation of and communication with the equipment.

\section{BEAM TESTS}

\section{Organisation, Intensities and Radiation}

The beam tests took place over two periods of about 48 hours, separated by two weeks (23/24 October and 6/7 November 2004). In preparation of the beam tests the dump situated just before the entrance to the LHC tunnel, some hundred meters away from the LHC injection point, was supplemented by temporary iron and concrete shielding. During the tests the complete underground area of LHC point 8 was closed, including about $300 \mathrm{~m}$ in the LHC tunnel towards point 1 and about $600 \mathrm{~m}$ in the LHC tunnel towards point 7 . The integrated and maximum beam intensities deposited on the beam dump had to be minimised in order to impact least on the areas further downstream where LHC installation was in full swing. For the same reason the beam tests were organised during weekends.

At the start of the test the energy of the TI 8 line was set to $449.2 \mathrm{GeV}$, following the energy calibrations done with ions in 2003 [3]. After the beam absorber at the top of TI 8 was moved out, the beam travelled all the way down the transfer line without the need for any beam threading. The picture of the first beam on the screen at the end of the line is shown in Fig. 4. It shows that the beam was rather well centred and that the optics was qualitatively correct.

For most of the TI 8 tests single pilot bunches were used, with an intensity of around $5 \cdot 10^{9}$ protons, or single bunches with higher intensity, around $3-4 \cdot 10^{10}$ protons, to improve the resolution of the beam diagnostics. For both weekends a total intensity between 5 and $15 \cdot 10^{13}$ protons was assumed beforehand. During the first weekend the integrated proton intensity reaching the end of TI 8 was $3.4 \cdot 10^{13}$ and for the second weekend $4.8 \cdot 10^{13}$ protons (Fig. 5).

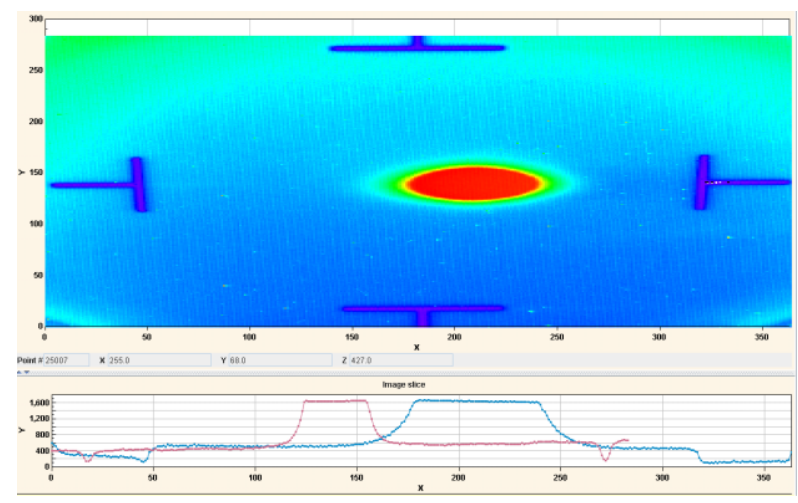

Figure 4: Screen image of the first beam which travelled down the TI 8 transfer line, taken on 23 October 2004.
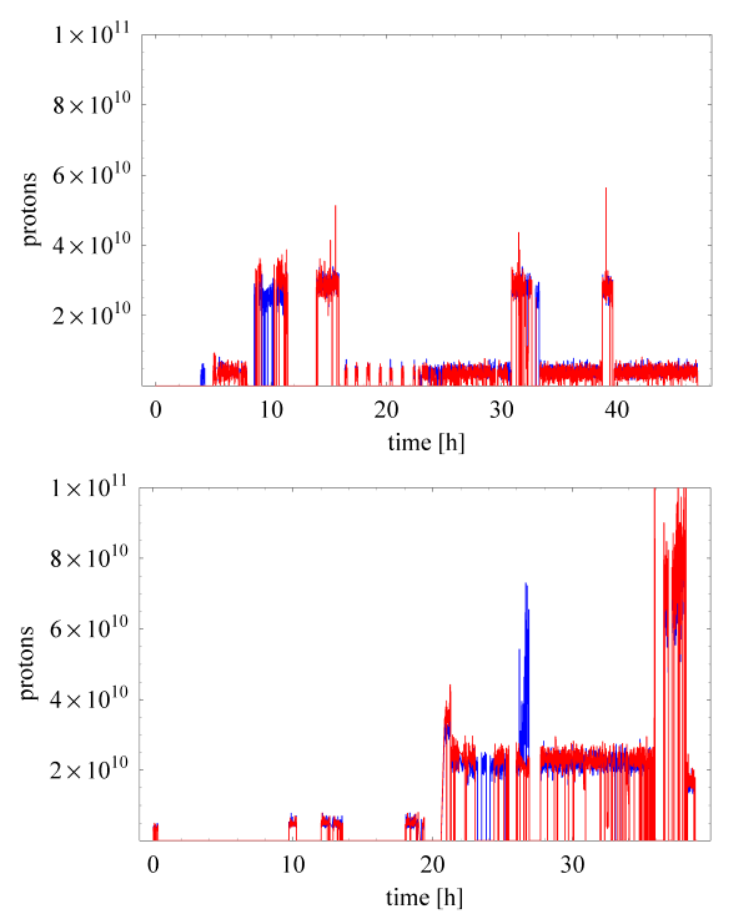

Figure 5: Intensities at the top of TI 8 (blue) and at the bottom of TI 8 (red) for the first (top) and second (bottom) weekend of beam tests.

The measured activity levels after beam operation were highest immediately after the second test period, with a maximum of $7 \mu \mathrm{Sv} / \mathrm{h}$ in the LHC tunnel just opposite of the beam dump [4]. This is explained by the relatively high beam intensities used at the end of the second test weekend (Fig. 5) while commissioning the beam instrumentation with multiple bunches. Additional shielding was installed at this position in the LHC tunnel for several days which allowed passing by without the need for a personal dosimeter. After both tests access was allowed to the LHC experimental cavern and the LHC tunnel a few hours after the beams in TI 8 were stopped with radiation levels not exceeding $0.5 \mu \mathrm{Sv} / \mathrm{h}$ at any place. 


\section{Optics Measurements}

An in-depth understanding of the LHC beam transfer lines is very important as the emittance budget of the LHC injector chain is very tight and less than about $5 \%$ for blow-up in the transfer process can be tolerated [5].

A large number of measurements was carried out. Only a brief summary is given here; more details can be found in other PAC05 contributions $[6,7,8,9]$.

The energy acceptance of $\pm 0.3 \%$ and the physical aperture, measured by producing oscillating trajectories and analysing the transmission, agree very well with particle tracking results and confirm the theoretical acceptance and physical aperture model [6].

The dispersion of TI 8 was measured during both test periods. The measurement during the first test weekend showed that 2 quadrupoles were set wrongly by about $20 \%$ due to a wrong Imax setting in a database. This error in the optics was also identified by the trajectory response measurements. This was corrected for the second test weekend, after which the optics model agreed very well with the measured dispersion and the trajectory response measurements [7].

Absolute measurements of the emittance and local Twiss parameters were made by combining the images of the 11 Optical Transition Radiation screens positioned along the line. The initial and final beam conditions have been calculated from these images, resulting in betatron mismatch factors between 10 and $20 \%$, which is very good for such a long and new transfer line [7].

The beam stability was measured to be better than $100 \mu \mathrm{m}$. According to expectations the major contribution to this displacement comes from the ripple of the extraction septum power converter. This could be traced back by using Model Independent Analysis on the data of many trajectories [8]. No significant correlation between the measured beam positions and the temperature of the magnets was found. The beam stability was confirmed by the results of the alignment procedure used for one collimator already installed in the upstream part of the line [9].

The coupling between the two planes was found to be smaller than $2 \%$ (amplitude coupling). The origin of this coupling was traced back to two quadrupoles suspected to be misaligned; a later alignment check of these elements did not confirm this [7].

\section{Instrumentation, Controls and Machine Protection}

The instrumentation, the control system as well as the installed part of the machine protection system performed excellently and largely contributed to the success of the TI 8 tests.

The overall good functioning of the comprehensive set of beam instrumentation equipment allowed a correct assessment of the beam. The sampling of the trajectory by the beam position monitoring system was found to be appropriate. The polarity of only a few pick-ups needed to be adjusted after calibration with beam. Both the upstream and downstream BCT in TI 8 were cross calibrated with beam against the SPS ring BCT.

With the TI 8 tests the control system also passed an important milestone. A full suite of applications and fixed displays, which will also be needed for the later LHC operation, was used to control and read out the equipment. The logging system and the associated data retrieval and viewing software performed well.

The good functioning of the Beam Interlock Controller was demonstrated. In contrast to the 2003 extraction tests, no interlocks needed permanent masking.

Before the LHC sector tests, planned for autumn 2006, TI 8 will need re-commissioning, including the last $100 \mathrm{~m}$ up to the injection point which are presently not yet installed. A number of machine studies are foreseen in 2006 to further deepen the present understanding of its optics with more detailed measurements.

\section{CONCLUSIONS}

The TI 8 beam tests constituted an important milestone and a first large-scale test-bed for hardware and concepts to be applied in the LHC. The basic design and operability of the transfer line have been confirmed. One can expect a good working transfer line, ready for the LHC sector test and LHC operation, delivering beam according to specification. Utmost care during construction and installation and thorough preparation of the beam tests during the hardware commissioning and the cold checkout periods contributed significantly to the successful beam tests.

\section{ACKNOWLEDGEMENTS}

The contributions of all colleagues involved in the design of the two beam lines, in the equipment construction and installation, the beam tests and the data analysis are gratefully acknowledged.

\section{REFERENCES}

[1] V.Mertens et al., "Status and Plans for the SPS to LHC Beam Transfer Lines TI 2 and TI 8", EPAC'04, Lucerne, July 2004.

[2] B.Goddard et al., "Beam Commissioning of the SPS LSS4 Extraction and the TT40 Transfer Line", EPAC'04, Lucerne, July 2004.

[3] J. Wenninger et al., "Energy Calibration of the SPS with Proton and Lead Ion Beam", these proceedings.

[4] H.Vincke, N.Conan, private communication.

[5] B.Goddard et al., "Expected Emittance Growth and Beam Tail Repopulation from Errors at Injection into the LHC", these proceedings.

[6] B.Goddard et al., "Aperture Studies of the SPS to LHC Transfer Lines", these proceedings.

[7] J.Wenninger et al., "Optics Studies of the LHC Beam Transfer Line TI 8", these proceedings.

[8] J.Wenninger et al., "Beam Stability of the LHC Beam Transfer Line TI 8", these proceedings.

[9] V.Kain et al., "Beam Based Alignment of the LHC Transfer Line Collimators", these proceedings. 